\title{
Lisa Herzog* \\ Basic Income and the Ideal of Epistemic Equality
}

DOI 10.1515/bis-2016-0009

Published online June 4, 2016

Abstract: In this short paper I explore whether a pro tanto argument for an unconditional basic income can be developed from a specific normative basis: the idea of epistemic equality as applied to organizations. In a broad sense, epistemic equality describes the idea that every individual has an equal right to speak up and to be heard if she has relevant information or knowledge to share, questions to ask, or complaints to bring forward. Epistemic equality has moral, instrumental, and instrumentally moral value in organizations. Drawing on Miranda Fricker's work on epistemic injustice (2009), I argue that we have good reasons to postulate epistemic equality as a normative ideal for organizations, even if - and precisely because they are otherwise hierarchically structured. The hypothesis I then proceed to explore is that an unconditional basic income, by providing better opportunities for "exit", might be a step that would strengthen epistemic equality in organizations.

Keywords: epistemic equality, unconditional basic income, basic income

\section{Introduction}

In this short paper I explore whether a pro tanto argument ${ }^{1}$ for an unconditional basic income can be developed from a specific normative basis: the idea of epistemic equality as applied to organizations. ${ }^{2}$ In a broad sense, epistemic equality describes the idea that every individual has an equal right to speak up and to be heard if she has relevant information or knowledge to share,

1 In particular, I do not discuss the normative issues around the legitimacy of wage labour as such, which would require a longer discussion than I can offer in the scope of this paper. By starting from an employment-related problem, my approach is necessarily "employmentcentric". I thank David Casassas for urging me to clarify this point.

2 I use the term "organizations" rather than "companies", because the issues I discuss are relevant for employees in public bureaucracies as well.

*Corresponding author: Lisa Herzog, Cluster "Normative Orders", Goethe University Frankfurt and Institut für Sozialforschung, Senckenberganlage 26, 60325 Frankfurt am Main, Germany, E-mail: herzog@em.uni-frankfurt.de 
questions to ask, or complaints to bring forward. Epistemic equality has moral, instrumental, and instrumentally moral value in organizations. Drawing on Miranda Fricker's work on epistemic injustice (2009), I argue that we have good reasons to postulate epistemic equality as a normative ideal for organizations, even if - and precisely because - they are otherwise hierarchically structured. The hypothesis I then proceed to explore is that an unconditional basic income, by providing better opportunities for "exit", might be a step that would strengthen epistemic equality in organizations. My arguments lead to a somewhat skeptical conclusion, however; what seems more promising than an unconditional basic income are more targeted measures that support those who suffer from epistemic injustices in organizations and, as I argue in concluding, cultural changes.

I hasten to add that these arguments do not amount to an all-thingsconsidered judgment on the idea of an unconditional basic income; nor do they provide a full picture of what morally justifiable organizations should look like. My reflections combine epistemic arguments and arguments from political philosophy, which is an intersection that does not receive as much theoretical attention as its practical relevance would suggest. What I find interesting about the perspective of epistemic equality is that it starts from a rather weak premise - a basic moral norm about mutual respect as bearers of knowledge - and, by incorporating empirical insights about the structure of organizations, $^{3}$ derives rather strong conclusions about the limits of organizational authority. How these can be institutionally realized, however, is a difficult issue and I cannot provide more than tentative hints in the conclusion of this paper.

\section{The ideal of epistemic equality}

The ideal of epistemic equality is a straightforward normative ideal: individuals should be considered as equals as bearers of knowledge. Epistemic equality is valuable in itself, but in the context of organizations, it has also instrumental and instrumentally moral value. Its moral value stems from the fact that human beings are moral equals, and that our status as bearers of knowledge is so intimately tied to our identity as rational and moral agents that the imperative of equal treatment translates into the sphere of knowledge as well. Miranda Fricker has recently explored the intrinsic connection between our identity as

3 To be sure, the empirical arguments I draw on may not always hold, but they are strong enough to allow plausible generalization. 
bearers of knowledge and the respect we owe one another as human beings. Not being taken seriously as a knower, she argues, violates the respect human beings owe one another:

To be wronged in one's capacity as a knower is to be wronged in a capacity essential to human value. When one is undermined or otherwise wronged in a capacity essential to human value, one suffers an intrinsic injustice. [...] We are long familiar with the idea, played out by the history of philosophy in many variations, that our rationality is what lends humanity its distinctive value. No wonder, then, that being insulted, undermined, or otherwise wronged in one's capacity as a giver of knowledge is something that can cut deep. (Fricker 2009, 44).

This does not mean that there cannot be any forms of unequal treatment of individuals as knowers - but these need to be justifiable. For example, in some situations there are good reasons to let those with greater expertise speak first, or to give one person the right to act as the moderator of a discussion in order to coordinate it better (see e. g. McMahon 1994, $86 \mathrm{ff}$. and $272 \mathrm{ff}$. on "E-authority" based on expertise and Chap. 8 on "C-authority" based on coordination). In practice, however, it is often difficult to delineate justifiable from unjustifiable forms of epistemic inequality. More specifically, in organizations epistemic equality can be threatened by asymmetries of power that are not justified by expertise or the need for coordination. Instead, they stem from the sheer fact that employees cannot easily "exit" an organization and have no right to "voice" either.

In addition to its moral value, epistemic equality can have instrumental value; understanding this instrumental value helps to see how it can also have instrumentally moral value. This instrumental value is especially important for individuals who have to cooperate in complex organizations that are larger than face-to-face communities. In such organizations, the relations between individuals are structured by hierarchies and often also by partly strategic relationships, for example because individuals compete for promotions. These two features can lead to serious distortions in the epistemic processes of organizations, for example because "the boss only gets the good news". But for organizations, especially for those operating in fast-changing and complex environments, it can be extremely important that all members openly share their knowledge with others, so that cues from the environment can be gathered and warning signals can be recognized early on. It is advantageous for them, therefore, to create an atmosphere in which all relevant knowledge is shared, no matter where someone stands in the organizational hierarchies - i. e. to develop practices of epistemic equality for instrumental reasons. This, however, is easier said than done: knowledge is something that people need to reveal voluntarily, 
and it is likely that they will only do so if they are treated fairly and with respect. A number of empirical studies suggest that fair reciprocity and a culture of respect matter for individuals to share their knowledge with organizations. ${ }^{4}$

One might object that such an instrumental perspective on epistemic equality fails to grasp its normative core and should play no role for normative considerations. But the instrumental processes in question can in turn have moral dimensions. A first possibility is that the goal of an organization, e. g. the care of patients in a hospital, has itself moral value, and that the instrumental value of epistemic equality receives instrumentally moral value from this goal. A second point, which applies to a far broader range of organizations than those whose goals are per se morally valuable, is that epistemic failures failures to acquire relevant knowledge, to pass it on, or to draw the right conclusions from it - can have disastrous consequences. Organizations are spaces of divided labor, in which interruptions of the chains of interrelated activities can have far-reaching consequences. Knowledge gaps, e.g. gaps about what has or has not been done, can lead to massive violations of basic moral norms, without anyone having evil intentions. To keep organizations, especially organizations that deal with risky technologies, morally "on track", one needs to keep their processes under control. The instrumental value of epistemic equality can gain moral weight if one of its aims is to prevent the violation of moral side constraints.

\section{Institutional levers for epistemic equality - can an unconditional basic income help?}

If one acknowledges that epistemic equality is an important normative ideal for organizations, whether for moral, instrumental, or instrumentally moral reasons, it suggests itself to ask about institutional levers that would promote it. Hence,

\footnotetext{
4 See e. g. Thibaut and Walker 1975, Folger 1977, Bies and Shapiro 1988 and Shapiro and Brett 2005 on the so-called "voice effect" that connects the possibility of raising one's voice and being heard to perceptions of organizational justice. Reversely, Pinder and Harlos 2001 find that "cultures of injustice" and "climates of silence" are antecendents of "employee silence". Tangirala and Ramanujam 2008 found that the variable "procedural justice climate" mediates individual-level antecedents of employee silence such as workgroup identification or professional commitment. Whiteside and Barclay 2013 found that transparent informational policies (as well as fairness, being taken seriously, and being able to express one's views) are significant antecedents of whether or not employees speak up with regard to a functional issue and a health and safety issue.
} 
one might suggest that an unconditional basic income could be a suitable candidate. One central argument in its favor is, after all, that it might help counterbalance the fundamental asymmetry between those who have to sell their labor in order to earn an income, and those who have an independent source of income (See e. g. Berry 2005, 212). By facilitating exit, an unconditional minimum income might also support epistemic equality in organizations: employees who know that they have a safety net in case they get fired might be more willing to speak up. They know that they will not end up without an income during the time it takes to find a new job or to start a self-employed business or a democratically run company (cf. similarly Blanc 2014, 484).

At second glance, however, one can raise a number of critical questions about whether an unconditional minimum income would really have these positive effects. Arguably, the dynamics of "exit" and "voice" can be more complex than this line of argument suggests. With regard to feasibility, supporters of an unconditional minimum income - and especially those who draw on the argument from epistemic equality - run into a dilemma: if an unconditional minimum income is low, it may be more feasible, but it may not have the desired effects. Individuals usually adapt their standard of living upwards to their current income, which is composed of the unconditional minimum income and their wage (and other forms of income or transfers they might receive). If, in the case of exit from their job, they fall back on the unconditional minimum income alone, they have to immediately cut back their spending, which can be so difficult that they do not perceive exit as an option they would take voluntarily.

Thus, to serve the purpose of strengthening epistemic equality, an unconditional minimum income would probably have to be quite high. This, however, makes questions of feasibility more urgent, and it also raises questions about ways in which this strategy might backfire. For example, it might make exit too easy, so that individuals lose any incentive to stay and speak up if they disagree with what is going on in an organization - and organizations could tell them to leave, instead of listening to what they have to say. Organizational life might become a very fleeting affair, with people coming and going in unpredictable ways. This might have certain normative advantages from an individual perspective, but it is hard to tell whether this is more likely to have a positive effect on epistemic equality in organizations, or whether it would turn their epistemic practices in a kind of "commons" that no-one feels responsible for.

Organizations might react to such tendencies by offering those employees whom they do not want to leave yet higher wages (cf. also Gintis \& Bowles 1993, 85). This, however, could exacerbate inequalities within organizations, creating an "in group" whose members are allowed to speak whenever they 
like, and an "out group" who are told to leave whenever they try to raise their voice. Some developments, for example the outsourcing of "non-essential" jobs into service companies, already go into the direction of creating different classes of employees with unequal rights. These developments are problematic not only from the perspective of epistemic equality, but, obviously, also from many other perspectives. One of the motives for promoting an unconditional minimum income is in fact to create stronger safety nets for those in precarious working conditions, but it is not clear what effects it would have for life within organizations. The overall case for an unconditional minimum income may be a strong one, but it seems too far-fetched to make an argument from epistemic equality, at least not before we do not have more empirical evidence of how an unconditional minimum income would change labour market dynamics.

For having a positive effect on epistemic equality in organizations, however, measures that support exit do not have to be unconditional. While an unconditional minimum income is a kind of "universal weapon" that is supposed to fight many social ills at once, one can also think about more targeted measures for supporting epistemic equality. Thus, there could be a specific kind of support for employees who are unemployed because they have raised their voice in an organization and are forced to leave. It could be temporary, because its aim would be only to bridge the gap until employees find a new job. In some cases, for example if someone goes public as a whistle-blower and does not find a job in the same industry any more, it might also be paid for a longer period, to allow the whistleblower to retrain for a different job. To be effective, it would have to be sufficiently high to make exit a real option for individuals; a pragmatic approach might be to use a certain percentage of someone's current income, with an upper threshold such that individuals in the higher echelons of the income scale who have sufficient savings do not receive it.

The idea for a support for "conscientious exit" is inspired by the advice given to business school graduates to have some "Go to hell money": money that allows them to say "go to hell" to anyone who wants them to do something they cannot reconcile with their own values (Gentile 2010, 78, quoting advice provided by a business school professor in a Harvard Business School case study). The advice holds that one should save a few months' income in order to feel secure in standing up for one's values. But for many employees this is very hard to do, especially in today's era of increasing inequality. It would be highly problematic if only those who can afford to safe sufficient money, or have other sources of support, can "afford" to speak up in organizations. Those with higher incomes for whom it is easier to save some "Go to hell money" are typically also those higher up in the hierarchical structures, and hence those for whom it is easier to speak up anyway. A specific measure for supporting 
those who could not otherwise afford to speak up, while certainly not solving the many other problems caused by income inequality, could at least help to curb its effects on epistemic practices in companies. ${ }^{5}$ Such a support system would probably not be too difficult to install, and the costs would presumably be relatively low, because it would function in large part by anticipation, and as a deterrent for companies, rather than actually being used.

Such support for "conscientious exit" would cover cases that result from the idiosyncrasies of specific organizations as well as cases that are more structural in nature, e. g. discrimination along lines of gender or race. Although it would probably not be sufficient to cure the latter kinds of problems, it would at least make sure that individuals do not have to endure them forever. Individuals would have an option that does not go as far as publicly blowing the whistle, which may not always be appropriate - and which is, in any case, a great risk for individuals - but which would still allow them to leave instead of being exposed to discrimination or social sanctions. By installing such a measure, public attention to problems of epistemic inequality could be raised, which might also have an encouraging effect on other individuals.

Such a targeted measure could also avoid a dynamic that might set in if a high unconditional minimum income were introduced: that organizations might tell individuals who insist on being heard to leave. This could be achieved by making public how many employees of an organization apply for support for “conscientious exit", e. g. on an annual basis. High numbers would, presumably, make companies look rather bad: they would suggest that they have problems with their epistemic culture. One might also publish different categories of cases - e. g. sexual discrimination, moral disagreement etc. - which would make clear what specific obstacles to epistemic equality exist in different companies. This would probably attract public attention, and thus create incentives for companies to address these specific problems. At the same time, the information generated by the statistics about such cases might also show where changes in the legal framework might be needed because companies fail to successfully address certain problems. ${ }^{6}$

5 Similar proposals have been made with regard to a "right to refuse" for functionaries (Bovens 1998, $181 \mathrm{ff}$.) and with regard to the protection of whistleblowers (ibid., $196 \mathrm{ff}$.).

6 Bovens similarly argues that one advantage of a "right to refusal" is epistemic: one receives valuable feedback about the organization's practices. He holds that providing an explanation should therefore be made "an obligatory part of the refusal procedure" $(1998,182)$. But this advantage could also be realized if the information thus generated is collected by a reliable committee, instead of being made public. 
To my knowledge, there is no real-life instantiation of such "conscientious exit support", which makes it hard to anticipate what other side-effects it might have. But there is one objection, at least, that one can rebut: that it would poison the atmosphere in organizations and destroy relationships of trust. This argument is invalid because the very reason for why such a measure might have a positive effect is that companies are social spaces in which trust and openness are difficult to establish, because of the existing inequalities of power and status. A support system for employees who leave because they are not listened to would restore some balance, and thereby hopefully also work towards more eye level-relationship and hence more trust. Those who worry about a genuinely trustful atmosphere in companies should therefore endorse, rather than reject, this argument.

\section{Conclusion}

In this paper, I have explored whether one can make a pro tanto argument for an unconditional minimum income based on the ideal of epistemic equality in organizations. My conclusion has been skeptical. An unconditional minimum income would probably have to be quite high to have the desired effect; in that case, however, it might backfire by changing the dynamics of labor markets in ways that are inimical to epistemic equality. As emphasized at the outset, this is not an all-things-considered argument; other arguments for an unconditional minimum income might outweigh these concerns. It might also be possible to counteract problematic effects on epistemic equality by other means, for example by an additional source of support for "conscientious exit".

I take it, however, that one of the general problems of finding institutional levers that support epistemic equality in organizations is that questions of voice and the right to be heard can be very hard to grasp in legal terms. One can be allowed to speak without being listened to. There can be subtle cultural norms, underneath the radar of legal rules, that discourage certain individuals in organizations from speaking up. There can also be forms of silencing or discrimination that are only visible to those directly involved, and hence difficult to bring to court, where hard evidence is required. Epistemic equality has a lot to do with a company's epistemic culture, and culture is, almost by definition, very hard to capture in legal terms.

Hence, I take it that in order to strengthen epistemic equality in organizations, we also need broader cultural changes with regard to the ways in which we conceptualise organizations and the relations within them. Human beings 
are what Taylor (1985) has called "self-interpreting animals": they interpret their own actions and those of others, and the concepts and images they draw on in these interpretive processes can have a deep impact on their thinking and their behavior. The accounts of organizations and labor markets that dominate in today's world push questions about epistemic equality and mutual respect, let alone other questions about morality, to the margins. They understand organizations in purely functional terms, as parts of a "system" that functions according to a logic of its own; usually, this is an economic logic, which is applied not only to business organizations but also, in increasing degrees, to public organizations. If individuals are seen as nothing but suppliers of "human capital", it is not surprising that their equal status as moral agents and as bearers of knowledge tends to be neglected.

While this picture of organizations may have heuristic value for certain theoretical questions, it is highly dangerous if it becomes self-fulfilling and individuals come to understand organizations as spaces in which even basic moral norms, such as the mutual respect of human beings as moral equals, have no place. Instead, we need to understand organizations as arenas in which these basic moral imperatives are as valid as in any other part of society. To be sure, different organizations have different tasks, and hence might be very different with regard to their structures, practices, and cultures but none of them should fall below a threshold of minimal moral norms. We should see them as falling into the scope of morality, and hold them accountable for behaving accordingly. This might lead to a cultural change that works towards more epistemic equality. Such a cultural change could also foster solidarity among different members of organizations, so that those with more power in organizations use it to fight for norms of epistemic equality that benefit those with less power.

The big question is, of course, how such cultural change can come about. I take it that there are various ways that would help implement it. One is the education of managers and of others who work in organizations, which could convey an account of organizations in which moral norms, including the norm of epistemic equality, are not marginalized any more. Another possibility are structural changes in the governance structures of organizations that might lead to a more egalitarian culture, for example forms of workplace democracy or workplace constitutionalism. And last but not least, cultural changes in the wider society would probably also help to change norms within organizations. From that perspective, there is one way in which an unconditional income might, after all, have a positive effect on epistemic equality in organizations: if it was perceived as an expression of the fact that we are all moral equals, who grant one another an unconditional minimum income out of solidarity with 
one another's common humanity, this would probably change the culture within organizations as well. In this way, it could also have a positive impact on egalitarian epistemic practices in organizations.

Acknowledgement: I would like to thank the audience at the workshop "Justice and Democracy in the Firm" at the University of Barcelona, and especially Serena Olsaretti as my commentator, for valuable feedback on the paper on which this piece is based. I would also like to thank David Casassas for inviting me to be part of this special issue and for helpful discussions.

\section{References}

Barry, B. (2005). Why social justice matters. Cambridge: Polity Press.

Bies, R. J., \& Shapiro, D. L. (1988). Voice and justification: Their influence on procedural fairness judgments. The Academy of Management Journal, 31(3), 676-685.

Blanc, S. (2014). Expanding workers' 'moral space': A liberal critique of corporate capitalism. Journal of Business Ethics, 120, 473-488.

Bovens, M. (1998). The quest for responsibility. Accountability and citizenship in complex organisations. Cambridge: Cambridge University Press.

Folger, R. (1977). Distributive and procedural justice: Combined impact of "voice" and improvement on experienced inequity. Journal of Personality and Social Psychology, 35, 108-119.

Fricker, M. (2009). Epistemic injustice: Power and the ethics of knowing. New York: Oxford University Press.

Gentile, M. C. (2010). Giving voice to values. How to speak your mind when you know what's right. New Haven/London: Yale University Press.

Gintis, S., \& Bowles, H. (1993). Power and wealth in a competitive capitalist economy. Philosophy \& Public Affairs, 21(4), 324-353.

McMahon, C. (1994). Authority and democracy. A general theory of government and management. Princeton, NJ: Princeton University Press.

Pinder, C. C., \& Harlos, K. P. (2001). Employee silence: Quiescence and acquiescence as responses to perceived injustice. In G. R. Ferris (ed.), Research in personnel and human resources management (Vol. 20, pp. 331-368). Greenwich, CT: JAI Press.

Shapiro, D. L., \& Brett, J. M. (2005). What is the role of control in organizational justice? In J. Greenberg, \& J. A. Colquitt, Handbook of Organizational Justice (pp. 155-177). Mahwah, NJ/London: Lawrence Erlbaum Associates.

Tangirala, S., \& Ramanujam, R. (2008). Employee silence on critical work issues: The cross level effects of procedural justice climate. Personnel Psychology, 61, 37-68.

Taylor, C. (1985). Self-interpreting animals. In Philosophical papers: Volume 1, human agency and language (pp. 45-76). Cambridge: Cambridge University Press.

Thibaut, J. W., \& Walker, L. 1975. Procedural justice: A psychological analysis. Hillsdale, NJ/New York: L. Erlbaum Associates.

Whiteside, D. B., \& Barclay, L. J. (2013). Echoes of silence: Employee silence as a mediator between overall justice and employee outcomes. Journal of Business Ethics, 116, 251-266. 